Review

\title{
Current status of the treatment of blood blister-like aneurysms of the supraclinoid internal carotid artery: A review
}

\author{
Tiefeng Ji1*, Yunbao Guo ${ }^{2 *}$, Xiuying Huang 3 , Baofeng $\mathrm{Xu}^{2}$, Kan $\mathrm{Xu}^{2}$, Jinlu $\mathrm{Yu}^{2 \bowtie}$ \\ 1. Department of Radiology, The First Hospital of Jilin University, Changchun, 130021, P.R. China; \\ 2. Department of Neurosurgery, The First Hospital of Jilin University, Changchun, 130021, P.R. China \\ 3. Department of Operation, The First Hospital of Jilin University, Changchun, 130021, P.R. China \\ *These authors contributed equally to this work.
}

$\triangle$ Corresponding author: Jinlu Yu, Department of Neurosurgery, The First Hospital of Jilin University, 71 Xinmin Avenue, Changchun 130021, China. Email: jlyu@jlu.edu.cn

(c) Ivyspring International Publisher. This is an open access article distributed under the terms of the Creative Commons Attribution (CC BY-NC) license (https://creativecommons.org/licenses/by-nc/4.0/). See http://ivyspring.com/terms for full terms and conditions.

Received: 2016.10.18; Accepted: 2017.02.26; Published: 2017.04.08

\begin{abstract}
Currently, the treatment of blood blister-like aneurysms (BBAs) of the supraclinoid internal carotid artery (ICA) is challenging and utilizes many therapeutic methods, including direct clipping and suturing, clipping after wrapping, clipping after suturing, coil embolization, stent-assisted coil embolization, multiple overlapping stents, flow-diverting stents, covered stents, and trapping with or without bypass. In these therapeutic approaches, the optimal treatment method for BBAs has not yet been defined based on the current understanding of BBAs of the supraclinoid ICA. Therefore, in this study, we aimed to review the literature from PubMed to discuss and analyze the pros and cons of the above approaches while adding our own viewpoints to the discussion. Among the surgical methods, direct clipping was the easiest method if the compensation of the collateral circulation of the intracranial distal ICA was sufficient or direct clipping did not induce stenosis in the parent artery. In addition, the clipping after wrapping technique should be chosen as the optimal surgical modality to prevent rebleeding from these lesions. Among the endovascular methods, multiple overlapping stents $(\geq 3)$ with coils may be a feasible alternative for the treatment of ruptured BBAs. In addition, flow-diverting stents appear to have a higher rate of complete occlusion and a lower rate of retreatment and are a promising treatment method. Finally, when all treatments failed or the compensation of the collateral circulation of the intracranial distal ICA was insufficient, the extracranial-intracranial (EC-IC) arterial bypass associated with surgical or endovascular trapping, a complex and highly dangerous method, was used as the treatment of last resort.
\end{abstract}

Key words: blood blister-like aneurysm, internal carotid artery, supraclinoid segment, treatment, review.

\section{Introduction}

Blood blister-like aneurysms (BBAs) account for $0.5-2.0 \%$ of ruptured intracranial aneurysms and lead to unusually high morbidity and mortality rates $[1,2]$. BBAs are small sidewall lesions that arise from nonbranched arteries. BBAs can grow in many sites, such as the supraclinoid internal carotid artery (ICA), middle cerebral artery, anterior communicating artery, basilar artery, and posterior cerebral artery $[3$,
4]. Among these sites, most BBAs arise from the supraclinoid ICA $[5,6]$. BBAs of the supraclinoid ICA are distinguished from saccular aneurysms by several unique characteristics, such as a hemispheric and broad-based appearance, a lack of an identifiable neck originating from a nonbranching site of an artery, instability, morphological changes on short-term angiographic follow-up, fragility and the tendency to 
rupture or regrow $[7,8]$. Therefore, the treatment methods for BBAs of the supraclinoid ICA, particularly ruptured BBAs, should be different from the treatments for saccular aneurysms [9].

Ruptured BBAs consist of a platelet plug covering a thin layer of adventitia, which overlies a defect in the intima and media that lacks the usual collagen layer; therefore, the management of BBAs of the supraclinoid ICA remains difficult [10]. BBAs should be treated as early as possible to avoid rebleeds [11]. However, until now, there has been no consensus as to how to treat BBAs of the supraclinoid ICA. During a literature review, we identified several techniques that have been applied to treat BBAs of the supraclinoid ICA, including direct clipping [12], suturing [13], clipping after wrapping [14, 15], the application of encircling clips [16], endovascular or surgical trapping with or without extra bypass [17, 18], coiling [19], stenting or flow-diverting stents [20] and multiple overlapping stents $[5,6]$.

Of these therapeutic approaches, the optimal treatment method for BBAs has not yet been defined based on the current understanding of BBAs of the supraclinoid ICA. Therefore, we herein review the literature to date. "Blood blister-like aneurysms" and "supraclinoid internal carotid artery aneurysms" were used as search terms of the "PubMed" and "Web of Science" databases to identify English-language literature. More than 200 articles were obtained, 83 of which are cited here. We aimed to review the literature from "PubMed" and "Web of Science" to discuss and analyze the pros and cons of the above approaches while adding our own viewpoints to the discussion.

\section{Classification and therapeutic protocol for BBAs}

Currently, the therapeutic protocol for BBAs of the supraclinoid ICA is rarely mentioned in the literature, and studies more often focus on specific therapeutic methods $[5,6]$. In fact, treatment of BBAs of the supraclinoid ICA abides by certain principles. In 2015, Bojanowski et al. proposed that BBAs of the supraclinoid ICA should be divided into four types. Type I BBAs are simply BBAs with a small bulge, whereas type II BBAs resemble a saccular aneurysm and involve part of the ICA wall. A small portion of the healthy artery wall must be included when type I and II BBAs are clipped. Type III BBAs involve a larger longitudinal portion of the ICA and this type of lesion requires the application of 2 clips in tandem. Type IV BBAs involve almost the entire ICA circumference, and the clipping after wrapping technique is required [21]. This classification provides some indications of the therapeutic options for treating BBAs based on their morphological features. Apparently, the morphological features are not sufficient. Han et al. commented that if the blood flow of the ICA is not considered, then the treatment of BBAs may be dangerous and cause forced trapping after intraoperative bleeding; therefore, neurosurgeons must be prepared to perform a bypass if needed [22].

Therefore, in addition to determining how to repair the BBAs, neurosurgeons should place more emphasis on the blood flow of the supraclinoid ICA when formulating a treatment plan for BBAs. We proposed a therapeutic protocol for BBAs of the supraclinoid ICA. Before treatment, the most important consideration is whether the therapeutic methods will reduce blood flow of the supraclinoid ICA. If the treatment will reduce blood flow, compensatory collateral circulation of the intracranial distal ICA should be evaluated. When the compensation is sufficient, all available surgical or endovascular treatments are options, even if they narrow or occlude the ICA. When the compensation is insufficient, a bypass between the external carotid artery (ECA) and ICA is necessary. Then, after bypass, trapping is a good treatment choice. Therapeutic methods that will not reduce blood flow in the ICA are alternative treatment options. However, during treatment, compensatory collateral circulation of the intracranial distal ICA should be immediately evaluated once the blood flow in the ICA decreases, perhaps using bypass.

Therefore, therapeutic approaches for BBAs can be classified into three types: (i) surgical treatments to obliterate the BBA combined with reconstruction of the supraclinoid ICA, including direct clipping and suturing, clipping after wrapping, and clipping after suturing, (ii) endovascular treatments to repair the BBAs combined with reconstruction of the supraclinoid ICA, including coiling, stenting, flow-diverting stents or multiple overlapping stents, and (iii) surgical or endovascular trapping assisted by extracranial-intracranial (EC-IC) arterial bypass. This study will document these therapeutic approaches in detail and provide some suggestions concerning BBA treatment.

\section{Surgical treatments for BBAs combined with reconstruction of the supraclinoid ICA}

\section{Direct clipping and suturing}

When BBAs are small and located at the dorsal supraclinoid ICA, they are treated with clips applied parallel to the ICA, which incorporate a thin band of tissue from the healthy arterial wall, without causing 
apparent arterial stenosis [21]. When BBAs are small and located at the ventral supraclinoid ICA, they are treated with clips applied perpendicular to the ICA or across the ICA, with the encircling clip applied to the medial artery wall $[23,24]$. When direct clipping is applied appropriately, good outcomes are achieved, without apparent ICA stenosis; for example, in 2009, Otani et al. reported a study of 4 patients who underwent direct clipping, 3 of whom did not present apparent supraclinoid ICA stenosis and achieved good outcomes [25]. In addition, in 2016, Pahl et al. also treated 4 patients with BBAs of the supraclinoid ICA using direct aneurysm clipping, 3 of whom did not present apparent supraclinoid ICA stenosis and achieved good outcomes [26].

Suturing the BBAs is also a good choice. For example, in 2009, Vashu et al. treated two patients with intraoperative rupture of BBAs of the supraclinoid ICA, and after suturing, these two patients showed normal caliber vessels with no recurrence of BBAs during the follow-up [27]. In addition, the repair of BBAs with vascular closure staple clips is a similar technique to suturing. For example, in 2004, Yanagisawa et al. reported a study of a patient with a ruptured BBA and discussed that several attempts to apply various clips and repair the wall with sutures failed to close the tear; finally, the large tear of the ICA was repaired using vascular closure staple clips, which were placed as close to the edges of the tear as possible to avoid vessel narrowing; therefore, this procedure is useful for the urgent repair of an aneurysm tear [28].

Although direct clipping and suturing are used treat BBAs without stenosis of the ICA, there is a risk of stenosis of the ICA during direct clipping or suturing [29], particularly for large BBAs such as type III BBAs, as proposed by Bojanowski et al. in 2015 [21]. In addition, the ICA may become narrow when multiple clips are used for large BBAs [30]. However, stenosis of the ICA does not always lead to clinical syndromes. The level of stenosis that does not result in clinical syndromes has not been clearly defined, but some studies have found that mild stenosis of the ICA is acceptable; for example, in 2006, Sim et al. reported that the direct clipping of six BBAs did not result in severe stenosis of the ICA and good outcomes were achieved [30]. In 2010, McLaughlin et al. reported the direct clipping of 7 BBAs of the supraclinoid ICA; although angiograms showed the expected mild stenosis of the ICA in 4 patients after clipping, no clinical or radiological cerebral infarctions were observed and the outcomes were favorable [31].

In fact, when neurosurgeons perform direct clipping or suturing of large BBAs, they may experience difficultly in controlling the clipping to avoid severe stenosis of the supraclinoid ICA $[25,29]$. Therefore, the preoperative balloon occlusion test or compression test should be performed to examine the collateral circulation. In 2015, Yu et al. reported on nine patients who underwent direct clipping of BBAs of the supraclinoid ICA, the blood flow in the middle cerebral artery decreased by less than $40 \%$ after the ICA was compressed, and no clinical symptoms were observed. Therefore, the patients were considered suitable for direct clipping of the BBA; although in some cases, the parent arteries developed severe stenosis, good outcomes were obtained [12]. Therefore, direct clipping requires that the compensation of the collateral circulation of the BBA side is sufficient to guarantee the safety of the operation.

Direct clipping and suturing are risky. In addition to severe stenosis of the supraclinoid ICA, the clip may be unstable and easily slip from the BBA; for example, in 1998, Abe et al. reported on a BBA case in which the clip slipped from the ICA after clipping, rebleeding occurred, and then the BBA was sutured [32]. In another example, in 2006, Sim et al. reported on two BBA cases where the clip slipped at the end of the dural closing and the BBAs had to be reclipped [30]. Cotton is often placed on the BBA before it is clipped to increase the friction force and prevent the clip from slipping [33]. In addition, during clipping or suturing, severe intraoperative bleeding may occur if a large tear occurs at the BBA; in this case, the bleeding cannot be controlled using direct clipping and suturing and BBA trapping becomes the treatment of last resort, which may also require a bypass [18, 34, 35]. Even when some BBAs are clipped, a temporary bypass with radial-middle cerebral artery was required to prevent ischemia [18]. The direct clipping methods with or without parent stenosis are shown in Figure 1A.

\section{Clipping after wrapping}

When BBAs of the supraclinoid ICA are large, such as the type III and IV BBAs proposed by Bojanowski et al. in 2015 [21]. Then direct clipping is difficult and dangerous because the wall of the lesion is so thin and fragile that the BBAs often rupture at the base during clipping; therefore, clipping after wrapping may be a good choice [8]. A strip of gauze is used to wrap the parent artery and BBA, and then both the gauze and BBA are clipped; this technique is known as clipping after wrapping [32]. The dorsal BBAs can be conveniently clipped using the clipping after wrapping method; however, when BBAs are located at the ventral wall of the supraclinoid ICA, a fenestrated clip is used, whereas a tiny piece of Teflon fiber and fibrin glue are used for effective adhesion of 
the margin [36]. In most cases, the clipping after wrapping technique was useful, particularly for cases without intraoperative rupture of the BBAs; for example, in 2006, Kubo et al. performed the clipping after wrapping technique with polytetrafluoroethylene on 6 BBAs of the ICA; all patients had an uneventful postoperative course, with no recurrent subarachnoid hemorrhage (SAH) during the follow-up period [37].

However, clipping after wrapping is risky; for example, in 1998, Abe et al. in 1998 on 2 patients who underwent clipping after wrapping, and in one case, the aneurysm had grown 17 days after the surgery,

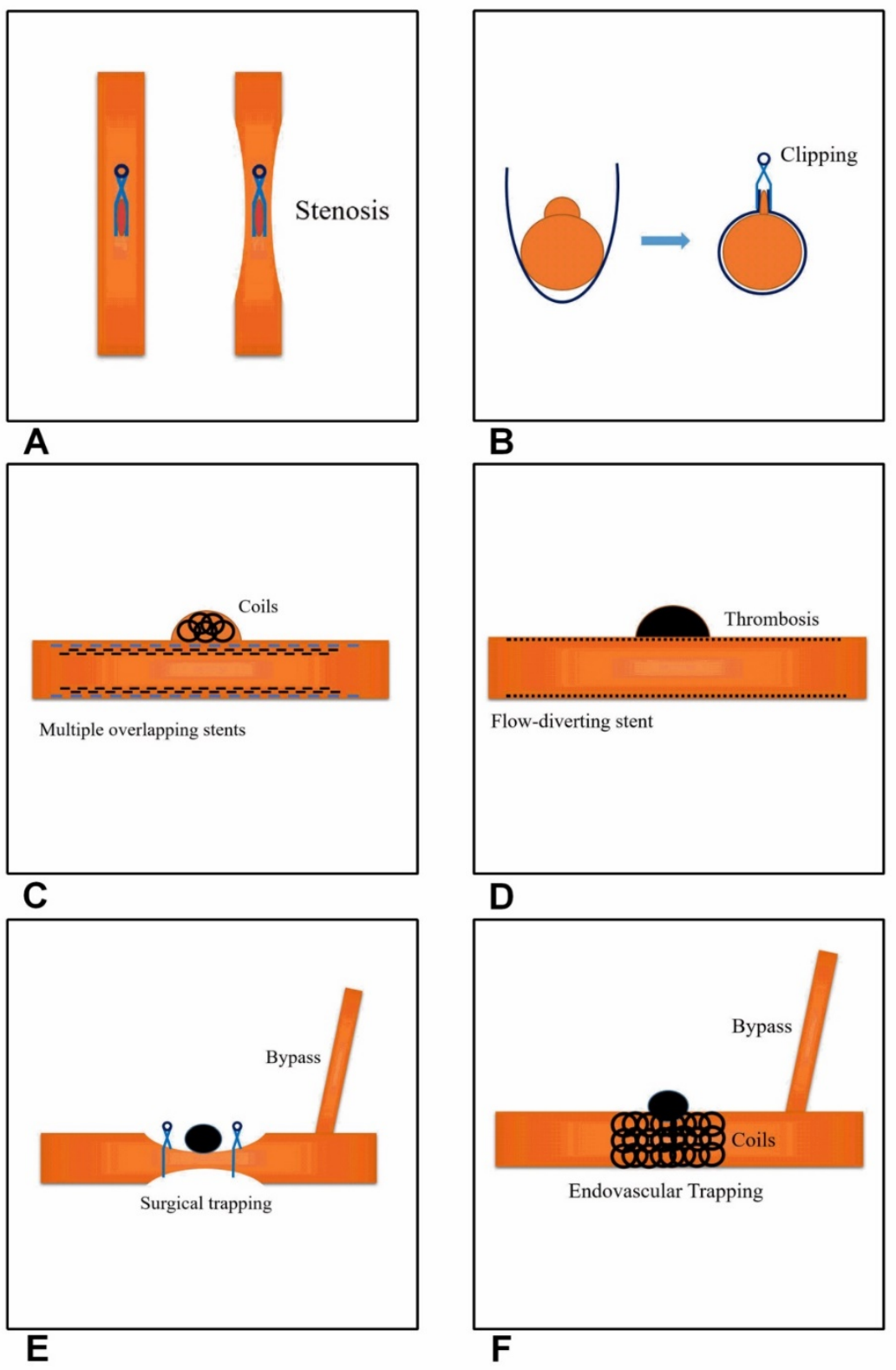

Figure 1. Current effective and feasible therapeutic methods for treating BBAs of the supraclinoid ICA. A, Direct clipping. The left image shows a parent artery without stenosis and the right image shows stenosis of the parent artery. B. Clipping after wrapping. C. Multiple overlapping stents with coiling. D, Flow-diverting stent application. E, Surgical trapping with EC-IC bypass. F, Endovascular trapping with EC-IC bypass. and in the other case, the patient experienced rebleeding on day 14 post-surgery [32]. An approach combining acute clipping after wrapping with subsequent definitive endovascular flow diversion reconstruction may improve the long-term treatment durability and effects [38]. In addition, stenosis of the ICA is possible; for example, in 2006, Sim et al. treated three patients using clipping combined with wrapping, one of whom exhibited left hemiparesis nine days later and moderate right ICA stenosis attributable to the two clips was observed in the immediate follow-up angiogram [30]. Intraoperative indocyanine green administration is helpful in preventing stenosis of the ICA when BBAs are treated with the clipping after wrapping technique [39].

The clipping after wrapping technique has been used as the optimal surgical modality to prevent rebleeding from BBA lesions because a large case study showed that this technique resulted in good outcomes; for example, in 2009, Lee et al. treated 15 consecutive patients with clipping after wrapping using cellulose fabric and good outcomes were achieved [14]. Various materials have been used for the wrapping, such as silastic sheets [40], cellulose fabric [14], polytetrafluoroethylene [37], and Bemsheets [39]. However, Gore-Tex is currently considered an excellent material for the circumferential wrapping of aneurysms and parent arteries because it is inert and does not cause tissue reactions or granuloma formation [41]. The method of clipping after wrapping is shown in Figure 1B.

\section{Clipping after suturing}

Although the clipping after wrapping technique is the optimal surgical method, it is favored in BBAs without intraoperative bleeding [25]. While neurosurgeons attempt to clip the BBA, the aneurysm can tear and produce a defect. If this occurs, then clipping after suturing can be performed if the defect is small, and this procedure should be safer than suturing alone. In 2016, Kantelhardt et al. reported on a 51-year-old woman with a BBA of the supraclinoid ICA and showed that the combination of micro-sutures and mini-clips for vessel wall adaptation, repair and the subsequent prevention of clip dislocation 
of the ruptured BBA was feasible and safe [42], particularly when associated with an encircling clip. In 2002, Yanaka et al. presented a case of clipping after suturing where the lesion was covered with Surgicel and fibrin glue, and then the temporary clip on the distal internal carotid artery was removed momentarily to allow retrograde blood flow and provide the counterforce necessary to maintain the vessel's tubular structure. An encircling clip was then applied to cover the entire circumference of the lesion, which resulted in a good outcome [16].

If the clipping after suturing technique is not sufficiently reliable, then the clipping after wrapping method with suturing technique is a good choice; for example, in 2006, Joo et al. reported on the successful treatment of two cases with direct suturing the arterial tear on the BBA, followed by circumferential wrapping with a silastic sheet and clip reinforcement, and both patients were discharged with no neurological deficits [40]. In another example, in 2009, Otani et al. reported on a 40-year-old woman with a BBA of the supraclinoid ICA who received clipping after suturing and achieved a good outcome [25]. Clipping after suturing requires only a short occlusion time for arterial repair, which helps avoid ischemic complications, but can result in ICA stenosis. For example, in 2014, Park et al. reported on a BBA located close to the anterior choroidal artery (AChA). Preserving the patency of the AChA was crucial after intraoperative rupture because the arterial defect was located very close to the AChA origin, and Direct clipping was difficult; therefore, suturing was performed and the suture line was reinforced using an aneurysm clip. However, the sutures produced severe stenosis of the ICA, but the patient achieved a good outcome because of the good collateral circulation [29].

\section{Endovascular treatment to repair the supraclinoid ICA}

Endovascular interventional techniques have evolved as an effective treatment for intracranial aneurysms [43]. Considerable interest has emerged regarding the use of endovascular approaches to treat BBAs [44]. In some studies, endovascular treatment of BBAs was associated with high rates of complete occlusion and good mid- to long-term neurological outcomes [45]. Various endovascular techniques have been applied to treat BBAs, such as coil embolization, stenting, stent-assisted coiling and flow-diverting stents [43].

\section{Stent-assisted coil embolization}

In the acute period, ruptured BBAs have a shallow sac and a very fragile neck, which makes simple coiling difficult and stent-assisted coil therapy necessary [46]. Moreover, the stent can provide the bridge by which vascular endothelial cells can cover the BBA neck [47]. In most cases, stent-assisted coil therapy is feasible; for example, in 2008, Korja et al. reported on patient with a BBA of the supraclinoid ICA who underwent stent-assisted coil embolization in the acute stage of SAH and the long-term follow-up outcome was good; therefore, stent-assisted coil embolization of ruptured BBAs in the acute stage appears to be a technically feasible treatment option [48]. If BBAs are treated in the late stage, they may be covered by a thick clot, which improves the stability of the BBA wall and leads to a better outcome. For example, in 2008, Doorenbosch et al. reported on a patient with a ruptured BBA. Two weeks after admission, the patient received stent-assisted coil embolization, there was no regrowth at the 6-month follow up, and the outcome was good [49].

For some BBAs, stent-assisted coil therapy may be difficult and have potential associated risks, including aneurysm neck disruption or distal coil migration. In these patients, stent-within-a-stent after stent-assisted coil therapy may provide additional support to the fragile aneurysm neck. For example, in 2007, Kim et al. first treated a patient with a BBA with Neuroform stent-assisted coil embolization and then a second stent was placed using the stent-within-a-stent technique to support the fragile aneurysm neck and decrease flow impingement; a follow-up digital subtraction angiography (DSA) performed 6 months after surgery demonstrated a radiolucent line crossing the aneurysm neck and suggested that the aneurysm neck was completely covered by a healing membrane [50]. The stent-within-a-stent technique is effective, particularly for recurrent BBAs [51]. For example, in 2009, Lee et al. treated 3 patients with recurrent BBAs who were initially treated with stent-assisted coil embolization by inserting a second stent using the stent-within-a-stent technique, and all 3 patients achieved excellent outcomes [2].

Although stent-assisted coil therapy can effectively treat BBAs in the acute period, the present stent-related endovascular technology is potentially hazardous due to intraoperative aneurysm rupture, rapid postoperative growth of the BBA and long-term recurrence of the BBA $[2,48,52,53]$. Intraoperative rebleeding was the most dangerous event and the causes included microcatheter or microwire perforation, stent deployment, systemic heparinization and antiplatelet application. For example, in 2008, Korja et al. reported on a BBA of the supraclinoid ICA where contrast extravasation from the BBA was identified during stent delivery and the stent released; thus, the operation had to be stopped 
[48]. Novel application of stent-assisted Onyx embolization may be helpful in avoiding intraoperative rebleeding [44]. Rapid growth and early rehemorrhage of the BBA after stent-assisted coil therapy is another problem; for example, in 2008, Ahn et al. described a BBA of the supraclinoid ICA that was treated with repeated coil embolization after the use of stent-assisted coiling due to the rapid growth of the BBA; therefore, stent-assisted coil embolization can be hazardous and careful serial follow-up angiography is necessary [52]. In some BBAs, there is no rapid growth stage, but rehemorrhage suddenly occurred; for example, in 2012, Ihn et al. performed primary stent-assisted coiling in 6 patients with BBAs, 2 of whom experienced early rehemorrhage, including one fatal SAH [53].

In addition, the stent-assisted coil embolization technique is associated with a high recurrence rate. In the report of 9 patients with BBAs by Lee et al., all the BBAs recurred after the initial treatment with stent-assisted coil embolization, and the patients required subsequent treatment with a second stent that was inserted with the stent-within-a-stent technique such that it covered the original stent placement and resulted in good outcomes [2]. Even stent-assisted coil embolization followed by stent-within-a-stent may result in recurrence of the BBAs; for example, Ihn et al. in a 2012 report showed that recurrence occurred in 2 BBAs after stent-assisted coil embolization followed by stent-within-a-stent and required complementary treatment, including repeated coil embolization and/or covered-stent placement [53]. In the 2013 study by Lim et al., 34 patients with ruptured BBAs underwent reconstructive treatment with stents and coils; the placement of a single stent with coils and a high Hunt and Hess grade were two independent risk factors for recurrence, particularly within 5 weeks after surgery [51]. In addition, the results of stent-assisted coil embolization are associated with the morphology of the BBA because ventral BBAs of the supraclinoid ICA tend to occlude progressively, even after an incomplete occlusion with stent-assisted coil embolization, whereas dorsal BBAs tend to recur and must be observed carefully [54].

In summary, reconstructive treatment with stents and coils appears to be a viable option for BBAs. Although stent-assisted coil embolization alone is an effective approach for treating BBAs, it is insufficient to prevent BBA regrowth. In contrast, stent-within-a-stent and covered stenting are effective at preventing rebleeding and BBA regrowth. Furthermore, in the 2016 by Song et al., the use multiple overlapping stents $(\geq 3)$ with coiling could be a feasible alternative for the treatment of ruptured
BBAs of the supraclinoid ICA, even in the era of flow-diverting stents; however, additional reports and follow-up are needed in a larger cohort [55]. The multiple overlapping stents with coiling method is shown in Figure 1C.

\section{Coil embolization}

Because BBAs of the supraclinoid ICA are very fragile and have a tendency to progress in size over a short period, primary coiling may be dangerous, given the lesion's wide neck, small size and weak wall that lacks collagenous tissue, particularly in the acute period [56]. Occasionally, when some patients waited for several weeks until the late period, the BBAs were treated with coil embolization because at this time, the ruptured BBAs may become spherical and covered by a thick clot, which eliminates the issue of wall fragility in this false aneurysm. For example, in 2004, Tanoue et al. treated a patient with a ruptured BBA of the supraclinoid ICA using coil embolization 40 days after admission, and the follow-up DSA demonstrated obliteration of the BBA; the author suggested that BBAs are unsuitable for surgery in the acute phase and that the application of coil embolization following progression of the lesion into a saccular aneurysm during the chronic stage may achieve good outcomes [19].

In another example, in 2006, Ezaki et al. treated a BBA of the supraclinoid ICA 13 days after the SAH and the DSA revealed that the shape and size of the BBA changed into a saccular aneurysm; on the 15th day, the author planned to treat the BBA using trapping with bypass, but the craniotomy revealed a thick thrombus covering the BBA; therefore, the author instead performed coil embolization on the 19th day and a good outcome was achieved, suggesting that coil embolization is an effective option for the management of selective cases of ruptured BBAs in the late period [57]. Unlike stent-assisted coil embolization, the best advantage of coil embolization of BBAs is that antiplatelet therapy is not needed and balloon-assisted coil embolization for wide-necked BBAs is feasible; for example, in 2011, Matsubara et al. reported on 9 patients with saccular-shaped ruptured BBAs, 7 of whom were treated with balloon-assisted coil embolization and 2 were embolized without balloon inflation, although a balloon catheter was placed at the ICA in the event that it was needed; moreover, three patients were treated in the acute period, two in the sub-acute period, and four in the chronic period; therefore, this method can be used to treat ruptured BBAs during the acute and sub-acute stages [58].

Because ruptured BBAs are a pseudoaneurysm covered with adventitia, immediate treatment is 
required once a ruptured BBA has been confirmed due to the high risk of rebleeding in a short time. Moreover, even in the chronic period, coil embolization of BBAs was more dangerous than for true saccular aneurysms because the BBA is not a true aneurysm [59]. In the 2011 report by Matsubara et al., one intraoperative rupture occurred, even with balloon assistance [58]. Therefore, coil embolization alone is not the optimal approach for treating ruptured BBAs.

\section{Multiple overlapping stents}

Theoretically, the placement of overlapping stents at the aneurysm neck diminishes the flow into the artery and the hemodynamic stress improves flow diversion and prevents rehemorrhage better than a single stent; furthermore, stents can induce thrombosis in BBAs that are refractory to coil embolization $[2,60]$. Therefore, the use of multiple overlapping stents may be a potentially effective treatment for BBAs; in 2014, Walsh et al. performed a single institution series and review of the literature and showed that the use of multiple overlapping stents as a monotherapy is a safe and effective strategy for treating the remodeling of BBAs arising from the supraclinoid ICA [54]. The multiple overlapping stents technique entails the deployment of longer and shorter stents; the longer stent is deployed first, which spans a large portion of the supraclinoid ICA segment and covers the aneurysm neck, whereas the shorter stent is then deployed within the lumen of the longer stent and also spans the aneurysm neck. Enterprise and Neuroform stents are good choices for this procedure; for example, in 2010, Gaughen et al. treated 6 patients with ruptured BBAs of the supraclinoid ICA using the stent-in-stent technique assisted by the application of Enterprise and Neuroform stents and no rebleeding was observed in any of the BBAs after the initial treatment [61].

The number stents required for an effective treatment is currently unclear, and two patients with BBAs of the supraclinoid ICA were treated in the 2006 report by Fiorella et al. - one was treated with the placement of a single Neuroform stent and the other was treated with the placement of 3 overlapping Neuroform stents; complete resolution of the BBA was achieved in both cases [60]. In another example in 2012, Ihn et al. reported on the treatment of a patient with a BBA of the supraclinoid ICA using double stenting and the late angiographic follow-up showed complete or progressive occlusion [53]. In addition, in 2011, Kim et al. reported on 3 cases of successful obliteration of BBAs of the supraclinoid ICA after the placement of multiple stents: 2 cases used 2 overlapping stents and 1 case used 3 overlapping stents. The follow-up DSA images of these 3 BBAs showed complete obliteration of the aneurysm; therefore, overlapping stents may reinforcing blood flow remodeling and arterial wall support, which may help reconstruct the fragile neck of the BBA and prevent its regrowth [62].

Although multiple overlapping stents are an effective treatment for BBAs of the supraclinoid ICA, clinicians have debated whether it is the optimal approach to resolve the BBAs. For example, in their study of 6 patients with BBAs, Gaughen et al. showed that only 3 patients presented no residual or recurrent aneurysm following the initial treatment and the other 3 patients required retreatment with coils after continued growth of the aneurysm, which was identified on follow-up angiography [61]. After the application of multiple overlapping stents, antiplatelet treatment may increase the risk of recurrence of BBAs of the supraclinoid ICA and postoperative rebleeding; therefore, antiplatelet medication was not administered before the procedure, but aspirin antiplatelet therapy was initiated immediately after the procedure was complete [62]. In addition, double antiplatelet treatment can lead to the refractory recurrence of BBAs; for example, in 2013, Chung et al. reported on a BBA of the supraclinoid ICA that recurred after being treated by the placement of five overlapping stents with coils, and after changing from double antiplatelet therapy (clopidogrel and aspirin) to a single antiplatelet therapy, spontaneous obliteration was achieved by thrombosis [63].

\section{Flow-diverting stents}

The treatment of BBAs of the supraclinoid ICA is associated with high morbidity and mortality; microsurgical clipping or wrapping and/or the use of traditional endovascular techniques to repair the lesion may result in frequent regrowth and rebleeds and, ultimately, high fatality rates [1]. Because of the purely endoluminal nature of arterial reconstruction, flow-diverting stents may represent an ideal option to repair ruptured BBAs [64]. A flow-diverting stent generally includes a silk flow diverter, a Pipeline embolization device, a surpass flow diverter, p64 and other new products, and these stents are self-extracting stent apparatuses with a high metal surface area (30-35\% metal coverage); after covering the neck of the aneurysm, the flow-diverting stent induces stasis, thrombosis, and regression of the aneurysm by disrupting the pulsatile flow into the aneurysm, which cures the aneurysm, and flow-diverting stents represent a revolutionary approach to the treatment of cerebral aneurysms, 
including BBAs of the supraclinoid ICA, particularly in patients who achieved unfavorable results and were initially managed using other endovascular treatment options [65-70].

The use of flow-diverting stents to treat ruptured BBAs is associated with high rates of complete occlusion and good long-term neurological outcomes in most patients; for example, in 2016, Linfante et al. treated 10 patients with ruptured BBAs of the supraclinoid ICA using a Pipeline embolization device, and the placement of a single Pipeline embolization device resulted in the immediate occlusion or near occlusion of the BBA in 9 of the 10 patients, and the follow-up DSA showed the complete occlusion of these BBAs [71]. In another example reported in 2015, Aydin et al. treated 8 patients with BBAs of the supraclinoid ICA using silk flow-diverting stents, 7 of whom achieved good outcomes [9]. In addition, in 2013 and 2014, Çinar et al., Chalouhi et al. and $\mathrm{Hu}$ et al. all reported similar results $[68,72,73]$.

Based on these results, repair of the ruptured BBAs with flow-diverting stents alone may be a safe and durable option. Generally, the use of a single flow-diverting stent is suitable for the treatment of a BBA, but multiple flow-diverting stents may be required; for example, in 2015, Nerva et al. treated a ruptured BBA of the supraclinoid ICA with two telescoping Pipeline embolization devices; however, an endoleak was noted between the two stents and was treated with balloon angioplasty, with good angiographic results [74]. In the 2015 report by Aydin et al., two flow-diverting stents were telescopically deployed in 3 patients, and the procedures were successful in all 3 patients and no acute complications developed [9]. In 2016, Hu et al. also reported similar results as Aydin et al. [73]. Therefore, multiple flow-diverting stents may be safely and effectively deployed when a single stent cannot occlude the blood flow into the BBA.

A dual antiplatelet regimen is needed when BBAs of the supraclinoid ICA are treated with flow-diverting stents to avoid thrombotic complications within the stent itself and in distal locations, such as in-stent thrombosis and thromboembolism, and all patients were administered dual antiplatelet therapy prior to placement of the flow-diverting stent [75]. However, dual antiplatelet therapy is associated with a risk of hemorrhagic complications, and the use dual antiplatelet therapy when a flow-diverting stent is implanted in an acutely ruptured BBA may affect surgical procedures such as extraventricular drain placement or delay surgery for a ventriculoperitoneal shunt, gastrostomy, tracheostomy, and decompressive craniectomy. Moreover, other relevant complications can occur, such as rupture of the BBA during the deployment of the flow-diverting stent [74]. Single or dual antiaggregant loading should be performed 6-8 $\mathrm{h}$ before treatment to reduce these complications and for a safer treatment [68]. In addition, the use of antiaggregant therapy to treat acute SAH should be modified for each patient due to the fragile nature of the recently ruptured BBA [76].

The most serious problem that occurs after the placement of flow-diverting stents is the continued existence or growth of the BBAs of the supraclinoid ICA. For example, in the 2016 report by Linfante et al., a BBA of the supraclinoid ICA remained patent and tended to grow, despite the placement of three flow-diverting stents in two procedures, but rerupture did not occur in this patient. However, the patient died of severe vasospasms, despite the administration of a dual antiplatelet regimen and growth of the lesion [71]. In addition, in 2015, Nerva et al. also reported on a similar case, where after the deployment of the first flow-diverting stent, the BBA of the supraclinoid ICA remained and enlarged; therefore, a second flow-diverting stent was placed, which overlapped the area of the aneurysm ostium covered by the first stent. However, the patient died of a diffuse infarction [74]. The causes of the continued existence or growth of BBAs after the application of flow-diverting stents vary and include mismatching of the stent, dual antiplatelet therapy and insufficient stent expansion; furthermore, insufficient stent expansion is an important cause because balloon angioplasty is required to open the stent [68]. Because the lack of immediate BBA occlusion of the supraclinoid ICA creates a risk of short-term re-rupture, more attention should be paid to this effect. The flow-diverting stent method for the treatment of ruptured BBAs is shown in Figure 1D.

\section{Covered stents}

Covered stents are used to treat BBAs or recurrent BBAs of the supraclinoid ICA; for example, in 2009, Lee et al. treated 3 patients with recurrent BBAs of the supraclinoid ICA who were initially treated with stent-assisted coil embolization with covered stents: two patients achieved excellent outcomes and complete occlusion of the BBA, whereas the remaining patient who received a covered stent died of ICA rupture during the procedure [2]. Therefore, covered stents should be used with caution because they have several limitations; for example, covered stents are far stiffer than bare stents, making their delivery into the intracranial ICA very difficult because the stiff stent may impinge on the fragile aneurysm neck during 
delivery and result in an unwanted outcome. In addition, covered stents may be too stiff to fully conform to the curved intracranial ICA wall, resulting in a failure to seal the aneurysm [77]. Additionally, BBAs are often located close to the origin of the posterior communicating artery and the anterior choroidal artery; thus, the use of a covered stent for treatment is less suitable.

\section{Trapping with or without bypass}

Generally, the treatments of BBAs of the supraclinoid ICA can be divided into reconstructive techniques with ICA preservation and deconstructive techniques with ICA sacrifice, which include trapping [45]. Trapping is an option for the treatment of BBAs and is associated with higher occlusion rates, particularly after the failure of initial endovascular treatments [78]. However, poor collateral circulation distal to the trapped ICA may lead to a higher risk of perioperative ischemic stroke and extremely poor outcomes. In the 2008 case-control study by Meling et al. that included 14 patients with BBAs of the supraclinoid ICA, 7 patients were treated with preservation of the ICA and 7 patients were treated with ICA trapping; of the 7 patients with ICA preservation, only 1 had a poor outcome, but cerebral infarcts developed in all the patients treated with ICA sacrifice, 2 of which occurred immediately after surgery and 5 occurred after a delay, with six of the patients dying and 1 surviving but presenting a poor condition [1].

Therefore, sacrifice of the ICA may lead to very poor outcomes, even in patients with adequate collateral capacity on preoperative angiograms, probably due to vasospasm-induced compromise of the cerebral collaterals [30]. Consequently, when trapping is considered, a balloon occlusion test for collateral flow must be performed prior to surgery and the external carotid artery should be examined to determine whether bypass surgery is required. In addition, cerebral vasospasm can affect ICA blood flow and should be considered. After trapping, the ipsilateral blood flow of the BBA depends on the collateral blood flow through the anterior communicating artery and ipsilateral posterior communicating artery; therefore, the collateral flow of the posterior communicating artery must be preserved $[18,30]$. If the collateral circulation of the distal ICA is sufficient, then single trapping of BBAs will result in good outcomes; in addition, the operation is not overly complex. However, if the collateral circulation is insufficient, EC-IC bypass may be needed.

Bypass is divided into high-flow and low-flow types, and high-flow bypass is currently the main type used. For example, in 2008, Başkaya et al. treated a series of 4 patients who presented with high-grade $\mathrm{SAH}$ due to a BBA; the patients were treated with high-flow EC-IC bypass, followed by trapping of the aneurysm, and the radial artery was used as the bypass conduit and good outcomes were achieved [78]. In 2009, Ishikawa et al. also reported on a series of 4 patients with ruptured BBAs of the ICA who were treated with EC-IC high-flow bypass, followed by inspection and trapping of the aneurysm; all patients were treated in the acute stage and excellent outcomes were achieved at the 3-month follow up [79]. In addition, in 2010, Kamijo et al. analyzed 7 consecutive patients with ruptured BBAs who were treated with trapping and EC-IC bypass during the acute $\mathrm{SAH}$ period, and the results showed that this surgical strategy might be a promising option for the treatment of BBAs during the acute period [80]. The acute phase was defined as less than $24 \mathrm{~h}$ [18]. Moreover, when performed prior to the onset of vasospasms, high-flow EC-IC bypass may prevent vasospasm-induced cerebral infarcts and the subsequent deaths associated with acute ICA sacrifice in SAH [1]. In some cases with relatively insufficient collateral circulation, low-flow bypass was sufficient. For example, in 2004, Islam et al. reported that a good outcome was achieved in a patient who experienced a ruptured BBA that was treated with coil embolization during the acute stage, followed by proximal occlusion in the chronic stage through a low-flow EC-IC bypass [81]. In another example from 2014, Kazumata et al. reported acceptable results in 2 patients with BBAs who were treated with ICA trapping and low-flow superficial temporal artery-middle cerebral artery bypass [18].

However, the treatment of BBAs with EC-IC bypass is associated with high risks and complications, which mainly included hand and brain ischemia, with ischemic hand complications possibly avoided by performing a preoperative Allen test; the measures used to reduce the risk of ischemic brain complications include careful back-bleeding and minimizing the temporary clipping time, and a wide Sylvian exposure will greatly reduce the need for retractors, and critical branches of the ICA should be preserved to prevent ischemia [82]. In addition, endovascular ICA trapping can be used to avoid surgical risks, such as surgical dissection and retraction of the edematous brain, and can be performed with balloon test occlusion. Staged trapping is a particularly good choice [78]. For example, in 2014, Kim et al. treated 11 consecutive patients with BBAs who underwent endovascular ICA trapping; subsequently, 3 patients underwent superficial temporal artery-middle cerebral artery 
bypass when balloon test occlusion revealed inadequate collateral circulation [17]. The merit of this staged treatment is its ability to prevent rerupture in the acute stage and to allow for proximal occlusion in the chronic stage, with or without an EC-IC bypass, after assessing the tolerance of the ICA occlusion. The EC-IC bypass method for treating BBAs is shown in Figure 1E and F.

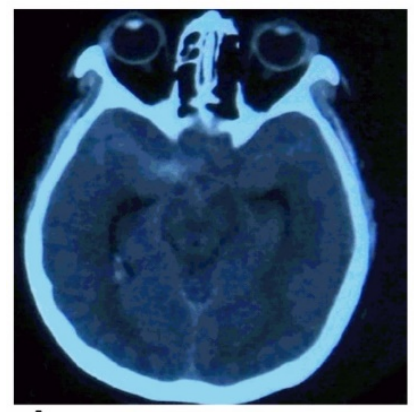

A

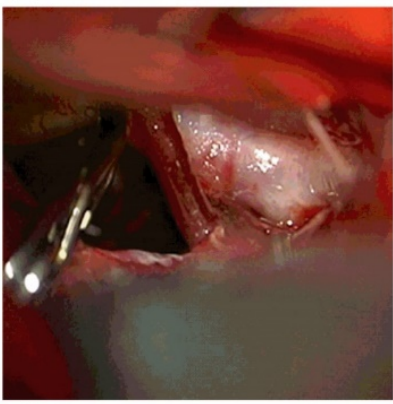

C

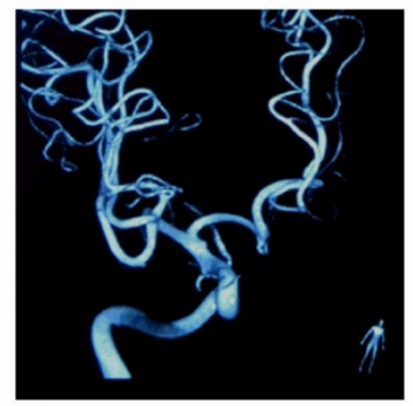

B

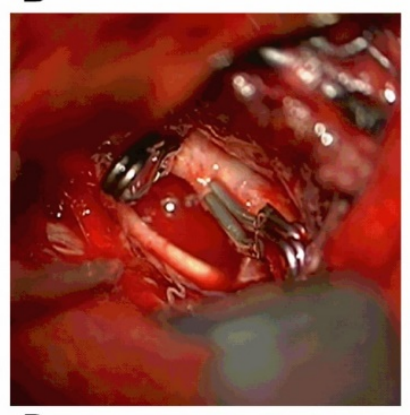

D

failed or the compensation by the collateral circulation of intracranial distal ICA was insufficient, then EC-IC bypass associated with surgical or endovascular trapping is used as the last resort, but the method is complex and highly risky.

At our institute, we prefer to perform the direct clipping operation because it is convenient and often results in a good outcome; a typical case is shown in Figure 2. In addition, profile visualized intraluminal support (LVIS) stents are a good choice due to the $12-21 \%$ surface area coverage [83], though double LVIS stents are sometimes necessary. The trapping with bypass method is still used as the last resort and is effective; a typical case is shown in Figure 3.

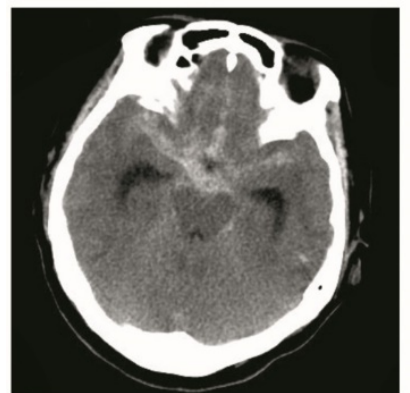

A

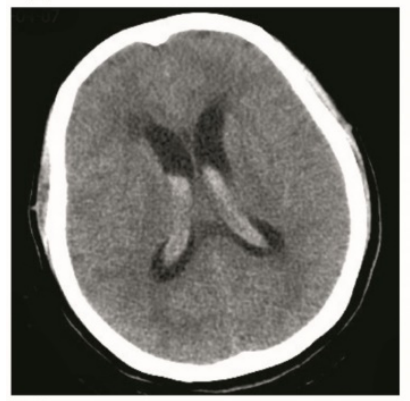

C

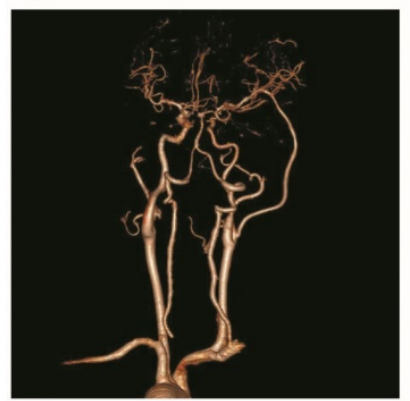

E

Figure 3. Trapping with bypass of BBAs of the supraclinoid ICA. A, CT of the first $\mathrm{SAH}$ showing the hemorrhage in the suprasellar cistern. $\mathrm{B}, \mathrm{CTA}$ showing a small BBA of the supraclinoid ICA. C, CT of the second SAH showing the intraventrical hemorrhage. D, DSA showing the BBA became bigger. E-F, Postoperative CTA showing that the BBA was trapped with EC-middle artery bypass. for ruptured BBAs of the supraclinoid ICA. In addition, flow-diverting stents appear to have a higher rate of complete occlusion and should be a promising treatment. Finally, when all treatments 
Table 1. Key points of each approach for BBAs

\begin{tabular}{|c|c|}
\hline Approaches & Key points \\
\hline $\begin{array}{l}\text { Direct clipping } \\
\text { and suturing }\end{array}$ & $\begin{array}{l}\text { The approach is feasible when BBAs are small and located at the ventral supraclinoid ICA. To guarantee the safety of the operation, the } \\
\text { approach may require that the compensation of the collateral circulation of the BBA side is sufficient. The approach was the easiest method if the } \\
\text { compensation of the collateral circulation of the intracranial distal ICA was sufficient or if the approach did not induce stenosis in the parent } \\
\text { artery. However, the clip may be unstable and easily slip from the BBA. In addition, during clipping or suturing, severe intraoperative bleeding } \\
\text { may occur if a large tear occurs at the BBA. }\end{array}$ \\
\hline $\begin{array}{l}\text { Clipping after } \\
\text { wrapping } \\
\text { Clipping after } \\
\text { suturing }\end{array}$ & $\begin{array}{l}\text { When BBAs are large, clipping after wrapping was feasible. Clipping after suturing is favored in BBAs with intraoperative bleeding, when the } \\
\text { aneurysm produced a defect. The clipping after wrapping technique can increase the stability of clipping and reduce the rupture risk of BBAs. } \\
\text { The approach has been used as the optimal surgical modality to prevent rebleeding from BBA lesions. However, clipping after wrapping and } \\
\text { clipping after suturing are risky, and the aneurysm may grow and rebleed after surgery. In addition, stenosis of the ICA after this method is } \\
\text { possible. }\end{array}$ \\
\hline $\begin{array}{l}\text { Stent-assisted } \\
\text { coil embolization } \\
\text { Coil } \\
\text { embolization }\end{array}$ & $\begin{array}{l}\text { If BBAs are treated in the late stage, coil embolization can be used because at this time, the ruptured BBAs may become spherical and covered by } \\
\text { a thick clot, which eliminates the issue of wall fragility in this false aneurysm. In most cases, stent-assisted coil therapy is feasible due to the } \\
\text { wide BBA neck. Moreover, the stent can provide the bridge by which vascular endothelial cells can cover the BBA neck. Although stent-assisted } \\
\text { coil embolization or coil embolization are effective approaches for treating BBAs, each is insufficient to prevent BBA regrowth. }\end{array}$ \\
\hline $\begin{array}{l}\text { Multiple } \\
\text { overlapping } \\
\text { stents }\end{array}$ & $\begin{array}{l}\text { When coiling embolization are refractory, the placement of overlapping stents diminishes hemodynamic stress, improves flow diversion and } \\
\text { prevents rehemorrhage. Furthermore, stents can induce thrombosis in BBAs. Multiple overlapping stents }(\geq 3) \text { with coils may be a feasible } \\
\text { alternative for the treatment of ruptured BBAs. After the application of multiple overlapping stents, antiplatelet treatment may increase the risk } \\
\text { of recurrence of BBAs and postoperative rebleeding. In addition, double antiplatelet treatment can lead to the refractory recurrence of BBAs. }\end{array}$ \\
\hline $\begin{array}{l}\text { Flow-diverting } \\
\text { stents }\end{array}$ & $\begin{array}{l}\text { When microsurgical clipping or wrapping and/or the use of traditional endovascular techniques to repair the lesion were difficult or resulted in } \\
\text { regrowth and rebleeds, the use of flow-diverting stents to treat ruptured BBAs was a good choice. Flow-diverting stents represent a } \\
\text { revolutionary approach to the treatment of cerebral aneurysms. However, dual antiplatelet therapy is associated with a risk of hemorrhagic } \\
\text { complications. Moreover, the most serious problem that occurs after the placement of flow-diverting stents is the continued existence or growth } \\
\text { of the BBAs. }\end{array}$ \\
\hline $\begin{array}{l}\text { Trapping with or } \\
\text { without bypass }\end{array}$ & $\begin{array}{l}\text { When all treatments failed or the compensation by the collateral circulation of intracranial distal ICA was insufficient, EC-IC bypass associated } \\
\text { with surgical or endovascular trapping was used as the last resort. Trapping is associated with higher occlusion rates. Because the trapping with } \\
\text { bypass is a complex technique, treatment of BBAs with EC-IC bypass is associated with high risks and complications, which mainly include } \\
\text { hand and brain ischemia. Accordingly, when trapping is considered, a balloon occlusion test for collateral flow must be performed prior to } \\
\text { surgery. }\end{array}$ \\
\hline
\end{tabular}

BBA: blood blister-like aneurysm; ICA: internal carotid artery; EC-IC: external carotid-internal carotid

\section{Competing Interests}

The authors have declared that no competing interest exists.

\section{References}

1. Meling TR, Sorteberg A, Bakke SJ, Slettebo H, Hernesniemi J, Sorteberg W. Blood blister-like aneurysms of the internal carotid artery trunk causing subarachnoid hemorrhage: treatment and outcome. J Neurosurg. 2008; 108: 662-71.

2. Lee BH, Kim BM, Park MS, Park SI, Chung EC, Suh SH, et al. Reconstructive endovascular treatment of ruptured blood blister-like aneurysms of the internal carotid artery. J Neurosurg. 2009; 110: 431-6.

3. Peschillo S, Miscusi M, Caporlingua A, Cannizzaro D, Santoro A, Delfini R, et al. Blister-like Aneurysms in Atypical Locations: A Single-Center Experience and Comprehensive Literature Review. World Neurosurg. 2015; 84: 1070-9.

4. Peschillo S, Missori P, Piano M, Cannizzaro D, Guidetti G, Santoro A, et al. Blister-like aneurysms of middle cerebral artery: a multicenter retrospective review of diagnosis and treatment in three patients. Neurosurg Rev. 2015; 38 : 197-202; discussion -3.

5. Szmuda T, Sloniewski P, Waszak PM, Springer J, Szmuda M. Towards a new treatment paradigm for ruptured blood blister-like aneurysms of the internal carotid artery? A rapid systematic review. J Neurointerv Surg. 2016; 8: 488-94.

6. Peschillo S, Cannizzaro D, Caporlingua A, Missori P. A Systematic Review and Meta-Analysis of Treatment and Outcome of Blister-Like Aneurysms. AJNR Am J Neuroradiol. 2016; 37: 856-61.

7. Sim SY, Chung J, Shin YS. Are blood blister-like aneurysms a specific type of dissection? A comparative study of blood blister-like aneurysms and ruptured mizutani type 4 vertebral artery dissections. J Korean Neurosurg Soc. 2014; 56 : 395-9.

8. Horie N, Morikawa M, Fukuda S, Hayashi K, Suyama K, Nagata I. Detection of blood blister-like aneurysm and intramural hematoma with high-resolution magnetic resonance imaging. J Neurosurg. 2011; 115: 1206-9.

9. Aydin K, Arat A, Sencer S, Hakyemez B, Barburoglu M, Sencer A, et al. Treatment of ruptured blood blister-like aneurysms with flow diverter SILK stents. J Neurointerv Surg. 2015; 7: 202-9.

10. Ogawa A, Suzuki M, Ogasawara K. Aneurysms at nonbranching sites in the surpaclinoid portion of the internal carotid artery: internal carotid artery trunk aneurysms. Neurosurgery. 2000; 47: 578-83; discussion 83-6.

11. Le Feuvre DE, Taylor AG. The management of very small/blister internal carotid artery aneurysms. Interv Neuroradiol. 2011; 17: 431-4.
12. Yu J, Xu B, Guo Y, Xu K. Direct clipping of a blister-like aneurysm in the supraclinoid segment of the internal carotid artery: a clinical analysis of nine cases. Int J Clin Exp Med. 2015; 8: 21786-95.

13. Regelsberger J, Matschke J, Grzyska U, Ries T, Fiehler J, Koppen J, et al. Blister-like aneurysms--a diagnostic and therapeutic challenge. Neurosurg Rev. 2011; 34: 409-16.

14. Lee JW, Choi HG, Jung JY, Huh SK, Lee KC. Surgical strategies for ruptured blister-like aneurysms arising from the internal carotid artery: a clinical analysis of 18 consecutive patients. Acta Neurochir (Wien). 2009; 151: 125-30.

15. Charbel FT, Gonzales-Portillo G, Hoffman W, Cochran E. Distal internal carotid artery pseudoaneurysms: technique and pitfalls of surgical management: two technical case reports. Neurosurgery. 1999; 45: 643-8; discussion 8-9.

16. Yanaka K, Meguro K, Nose T. Repair of a tear at the base of a blister-like aneurysm with suturing and an encircling clip: technical note. Neurosurgery. 2002; 50: 218-21.

17. Kim BC, Kwon OK, Oh CW, Bang JS, Hwang G, Jin SC, et al. Endovascular internal carotid artery trapping for ruptured blood blister-like aneurysms: long-term results from a single centre. Neuroradiology. 2014; 56: 211-7.

18. Kazumata K, Nakayama N, Nakamura T, Kamiyama H, Terasaka S, Houkin K. Changing treatment strategy from clipping to radial artery graft bypass and parent artery sacrifice in patients with ruptured blister-like internal carotid artery aneurysms. Neurosurgery. 2014; 10 Suppl 1: 66-72; discussion 3.

19. Tanoue S, Kiyosue H, Matsumoto S, Yamashita M, Nagatomi H, Mori H. Ruptured "blisterlike" aneurysm with a pseudoaneurysm formation requiring delayed intervention with endovascular coil embolization. Case report. J Neurosurg. 2004; 101: 159-62.

20. Rasskazoff S, Silvaggio J, Brouwer PA, Kaufmann A, Nistor A, Iancu D. Endovascular treatment of a ruptured blood blister-like aneurysm with a flow-diverting stent. Interv Neuroradiol. 2010; 16: 255-8.

21. Bojanowski MW, Weil AG, McLaughlin N, Chaalala C, Magro E, Fournier JY. Morphological aspects of blister aneurysms and nuances for surgical treatment. J Neurosurg. 2015; 123: 1156-65.

22. Han Z, Qi H, Yin W, Du Y. Letter to the Editor: Low-flow bypass and wrap-clipping for ruptured blister aneurysms of the ICA. J Neurosurg. 2016; 124: $1143-4$.

23. Tekkok IH, Bakar B. Ruptured blister-like aneurysm of distal internal carotid artery: a distinct entity. Turk Neurosurg. 2008; 18: 439-45.

24. Sekula RF, Jr., Cohen DB, Quigley MR, Jannetta PJ. Primary treatment of a blister-like aneurysm with an encircling clip graft: technical case report. Neurosurgery. 2006; 59: ONSE168; discussion ONSE.

25. Otani N, Takasato Y, Masaoka H, Hayakawa T, Yoshino Y, Yatsushige H, et al. Clinical and radiological findings and surgical management of ruptured aneurysms at the non-branching sites of the internal carotid artery. J Clin Neurosci. 2009; 16: 1018-23. 
26. Pahl FH, de Oliveira MF, Teles Gomes Mde Q, Capel Cardoso AC, Rotta JM. Blister-Like Aneurysms: Report of Successful Surgical Treatment of Consecutive Cases and Review of the Literature. World Neurosurg. 2016; 89: 376-81.

27. Vashu R, Tan S, Wong AS. Microsuture repair of intra-operative ruptures of cerebral aneurysms of the internal carotid artery. J Clin Neurosci. 2009; 16: 960-2.

28. Yanagisawa T, Mizoi K, Sugawara T, Suzuki A, Ohta T, Higashiyama N, et al. Direct repair of a blisterlike aneurysm on the internal carotid artery with vascular closure staple clips. Technical note. J Neurosurg. 2004; 100: 146-9.

29. Park J. Blood blister-like aneurysm with rupture point close to origin of anterior choroidal artery. J Korean Neurosurg Soc. 2014; 56: 500-3.

30. Sim SY, Shin YS, Cho KG, Kim SY, Kim SH, Ahn YH, et al. Blood blister-like aneurysms at nonbranching sites of the internal carotid artery. J Neurosurg. 2006; 105: 400-5

31. McLaughlin N, Laroche M, Bojanowski MW. Surgical management of blood blister-like aneurysms of the internal carotid artery. World Neurosurg. 2010; 74: 483-93.

32. Abe M, Tabuchi K, Yokoyama H, Uchino A. Blood blisterlike aneurysms of the internal carotid artery. J Neurosurg. 1998; 89: 419-24.

33. Mooney MA, Kalani MY, Nakaji P, Albuquerque FC, McDougall CG, Spetzler $\mathrm{RF}$, et al. Long-term Patient Outcomes After Microsurgical Treatment of Blister-Like Aneurysms of the Basilar Artery. Neurosurgery. 2015; 11 Suppl 3: 387-93.

34. Joo S, Kang MH, Lim T, Lee SK, Hwang HS. Iatrogenic rupture of undiagnosed blood blister-like aneurysm during aneurysmal neck clipping. Korean J Anesthesiol. 2014; 67: S108-10.

35. Kawashima A, Okada Y, Kawamata T, Onda H, Kubo O, Hori T. Successful treatment of a blood blister-like aneurysm of the internal carotid artery by trapping with a high-flow bypass. J Clin Neurosci. 2008; 15: 797-800.

36. Kurokawa $Y$, Wanibuchi $M$, Ishiguro $M$, Inaba $K$. New method for obliterative treatment of an anterior wall aneurysm in the internal carotid artery: encircling silicone sheet clip procedure--technical case report. Neurosurgery. 2001; 49: 469-72

37. Kubo Y, Ogasawara K, Tomitsuka N, Otawara Y, Watanabe M, Ogawa A. Wrap-clipping with polytetrafluoroethylene for ruptured blisterlike aneurysms of the internal carotid artery. Technical note. J Neurosurg. 2006; 105: 785-7.

38. Kalani MY, Albuquerque FC, Levitt M, Nakaji P, Spetzler RF, McDougall C. Pipeline embolization for definitive endoluminal reconstruction of blister-type carotid aneurysms after clip wrapping. J Neurointerv Surg. 2016; 8: 495-500.

39. Han MS, Joo SP, Jung SH, Kim TS. Specific and helpful intraoperative indocyanine green videoangiography finding of blood blister-like aneurysm of internal carotid artery. Acta Neurochir (Wien). 2015; 157: 1849-54

40. Joo SP, Kim TS, Moon KS, Kwak HJ, Lee JK, Kim JH, et al. Arterial suturing followed by clip reinforcement with circumferential wrapping for blister-like aneurysms of the internal carotid artery. Surg Neurol. 2006; 66: 424-8; discussion 8-9.

41. Safavi-Abbasi S, Moron F, Sun H, Wilson C, Frock B, Oppenlander ME, et al. Techniques and Outcomes of Gore-Tex Clip-Wrapping of Ruptured and Unruptured Cerebral Aneurysms. World Neurosurg. 2016; 90: 281-90.

42. Kantelhardt SR, Archavlis E, Giese A. Combined suture and clipping for the reconstruction of a ruptured blister-like aneurysm. Acta Neurochir (Wien). 2016.

43. Molyneux AJ, Birks J, Clarke A, Sneade M, Kerr RS. The durability of endovascular coiling versus neurosurgical clipping of ruptured cerebral aneurysms: 18 year follow-up of the UK cohort of the International Subarachnoid Aneurysm Trial (ISAT). Lancet. 2015; 385: 691-7.

44. Ashour R, Dodson S, Aziz-Sultan MA. Endovascular management of intracranial blister aneurysms: spectrum and limitations of contemporary techniques. J Neurointerv Surg. 2016; 8: 30-7.

45. Rouchaud A, Brinjikji W, Cloft HI, Kallmes DF. Endovascular Treatment of Ruptured Blister-Like Aneurysms: A Systematic Review and Meta-Analysis with Focus on Deconstructive versus Reconstructive and Flow-Diverter Treatments. AJNR Am J Neuroradiol. 2015; 36: 2331-9.

46. Yu B, Zheng J, Hong Y, Chen L, Xi Z, Yu M, et al. Stent-Assisted Coil Embolization of Ruptured Supraclinoid Blood Blister-Like Aneurysm of Internal Carotid Artery. Turk Neurosurg. 2016; 26: 219-22.

47. Starke RM, Turk A, Ding D, Crowley RW, Liu KC, Chalouhi N, et al. Technology developments in endovascular treatment of intracranial aneurysms. J Neurointerv Surg. 2016; 8: 135-44.

48. Korja M, Rautio R, Valtonen S, Haapanen A. Primary treatment of ruptured blood blister-like aneurysms with stent-assisted coil embolization: report of two cases. Acta Radiol. 2008; 49: 180-3.

49. Doorenbosch $X$, Harding $M$. Primary treatment of a blood-blister-like aneurysm of the internal carotid artery with Guglielmi detachable coil embolisation. J Clin Neurosci. 2008; 15: 1276-9.

50. Kim BM, Chung EC, Park SI, Choi CS, Won YS. Treatment of blood blister-like aneurysm of the internal carotid artery with stent-assisted coil embolization followed by stent-within-a-stent technique. Case report. J Neurosurg. 2007; 107: 1211-3

51. Lim YC, Kim BM, Suh SH, Jeon P, Kim SH, Ihn YK, et al. Reconstructive treatment of ruptured blood blister-like aneurysms with stent and coil. Neurosurgery. 2013; 73: 480-8.
52. Ahn JY, Cho JH, Jung JY, Lee BH, Yoon PH. Blister-like aneurysms of the supraclinoid internal carotid artery: challenging endovascular treatment with stent-assisted coiling. J Clin Neurosci. 2008; 15: 1058-61.

53. Ihn YK, Kim SH, Sung JH, Kim TG. The efficacy of endovascular treatment of ruptured blood blister-like aneurysms using stent-assisted coil embolization. Interv Neuroradiol. 2012; 18: 432-41.

54. Walsh KM, Moskowitz SI, Hui FK, Spiotta AM. Multiple overlapping stents as monotherapy in the treatment of 'blister' pseudoaneurysms arising from the supraclinoid internal carotid artery: a single institution series and review of the literature. J Neurointerv Surg. 2014; 6: 184-94.

55. Song J, Oh S, Kim MJ, Chung J, Lim YC, Kim BS, et al. Endovascular treatment of ruptured blood blister-like aneurysms with multiple $(>/=3)$ overlapping Enterprise stents and coiling. Acta Neurochir (Wien). 2016; 158: 803-9.

56. Vajkoczy P. Editorial on a paper entitled "Combined suture and clipping for the reconstruction of a ruptured blister-like aneurysm". Acta Neurochir (Wien). 2016; 158: 1913-5

57. Ezaki Y, Takahata H, Kamada K, Baba S, Kaminogo M. Aneurysmal embolization of a blisterlike aneurysm of the internal carotid artery: a case report and review of the literature. Surg Neurol. 2006; 65: 628-30; discussion 30 .

58. Matsubara N, Miyachi S, Tsukamoto N, Izumi T, Naito T, Haraguchi K, et al. Endovascular coil embolization for saccular-shaped blood blister-like aneurysms of the internal carotid artery. Acta Neurochir (Wien). 2011; 153: 287-94.

59. Haji FA, Boulton MR, de Ribaupierre S. Blister-like supraclinoid internal carotid artery pseudoaneurysm in a 15-year-old male: case report and review of the literature. Pediatr Neurosurg. 2011; 47: 449-54.

60. Fiorella D, Albuquerque FC, Deshmukh VR, Woo HH, Rasmussen PA, Masaryk TJ, et al. Endovascular reconstruction with the Neuroform stent as monotherapy for the treatment of uncoilable intradural pseudoaneurysms. Neurosurgery. 2006; 59: 291-300; discussion 291-300.

61. Gaughen JR, Jr., Hasan D, Dumont AS, Jensen ME, McKenzie J, Evans AJ. The efficacy of endovascular stenting in the treatment of supraclinoid internal carotid artery blister aneurysms using a stent-in-stent technique. AJNR Am J Neuroradiol. 2010; 31: 1132-8.

62. Kim YW, Park IS, Baik MW, Jo KW. Endovascular treatment of blood blister-like aneurysms using multiple self-expanding stents. J Korean Neurosurg Soc. 2011; 49: 116-9.

63. Chung J, Kim BM, Lim YC. Five overlapping enterprise stents in the internal carotid artery-to-middle cerebral artery to treat a ruptured blood blister-like aneurysm. Neurol Sci. 2013; 34: 1485-7.

64. Heiferman DM, Billingsley JT, Kasliwal MK, Johnson AK, Keigher KM, Frudit $\mathrm{ME}$, et al. Use of flow-diverting stents as salvage treatment following failed stent-assisted embolization of intracranial aneurysms. J Neurointerv Surg. 2015.

65. Colby GP, Lin LM, Huang J, Tamargo RJ, Coon AL. Utilization of the Navien distal intracranial catheter in 78 cases of anterior circulation aneurysm treatment with the Pipeline embolization device. J Neurointerv Surg. 2013; 5 Suppl 3: iii16-21.

66. Chalouhi N, Tjoumakaris S, Phillips JL, Starke RM, Hasan D, Wu C, et al. A single pipeline embolization device is sufficient for treatment of intracranial aneurysms. AJNR Am J Neuroradiol. 2014; 35: 1562-6.

67. Fischer S, Aguilar-Perez M, Henkes E, Kurre W, Ganslandt O, Bazner H, et al. Initial Experience with p64: A Novel Mechanically Detachable Flow Diverter for the Treatment of Intracranial Saccular Sidewall Aneurysms. AJNR Am J Neuroradiol. 2015; 36: 2082-9.

68. Cinar C, Oran I, Bozkaya H, Ozgiray E. Endovascular treatment of ruptured blister-like aneurysms with special reference to the flow-diverting strategy. Neuroradiology. 2013; 55: 441-7.

69. Consoli A, Nappini S, Renieri L, Limbucci N, Ricciardi F, Mangiafico S. Treatment of two blood blister-like aneurysms with flow diverter stenting. J Neurointerv Surg. 2012; 4: e4

70. Princiotta C, Dall'olio M, Cirillo L, Leonardi M. Staged treatment of a blood blister-like aneurysm with stent-assisted coiling followed by flow diverter in-stent insertion. A case report. Interv Neuroradiol. 2011; 17: 365-70.

71. Linfante I, Mayich M, Sonig A, Fujimoto J, Siddiqui A, Dabus G. Flow diversion with Pipeline Embolic Device as treatment of subarachnoid hemorrhage secondary to blister aneurysms: dual-center experience and review of the literature. J Neurointerv Surg. 2016

72. Chalouhi N, Zanaty M, Tjoumakaris S, Gonzalez LF, Hasan D, Kung D, et al. Treatment of blister-like aneurysms with the pipeline embolization device. Neurosurgery. 2014; 74: 527-32; discussion 32.

73. Hu YC, Chugh C, Mehta H, Stiefel MF. Early angiographic occlusion of ruptured blister aneurysms of the internal carotid artery using the Pipeline Embolization Device as a primary treatment option. J Neurointerv Surg. 2014; 6: $740-3$

74. Nerva JD, Morton RP, Levitt MR, Osbun JW, Ferreira MJ, Ghodke BV, et al. Pipeline Embolization Device as primary treatment for blister aneurysms and iatrogenic pseudoaneurysms of the internal carotid artery. J Neurointerv Surg. 2015; 7: 210-6

75. Lv X, Yang H, Liu P, Li Y. Flow-diverter devices in the treatment of intracranial aneurysms: A meta-analysis and systematic review. Neuroradiol J. 2016; 29: 66-71.

76. Meckel S, Singh TP, Undren P, Ramgren B, Nilsson OG, Phatouros C, et al. Endovascular treatment using predominantly stent-assisted coil embolization 
and antiplatelet and anticoagulation management of ruptured blood blister-like aneurysms. AJNR Am J Neuroradiol. 2011; 32: 764-71.

77. Wang G, Zhang GZ, Li MZ, He XY, Liu D, Song Y, et al. [Efficacy and safety of Willis covered stent for treatment of blood blister-like aneurysm]. Nan Fang Yi Ke Da Xue Xue Bao. 2016; 36: 1165-8.

78. Baskaya MK, Ahmed AS, Ates O, Niemann D. Surgical treatment of blood blister-like aneurysms of the supraclinoid internal carotid artery with extracranial-intracranial bypass and trapping. Neurosurg Focus. 2008; 24: E13.

79. Ishikawa T, Mutoh T, Nakayama N, Yasuda H, Nomura M, Kazumata K, et al. Universal external carotid artery to proximal middle cerebral artery bypass with interposed radial artery graft prior to approaching ruptured blood blister-like aneurysm of the internal carotid artery. Neurol Med Chir (Tokyo). 2009; 49: 553-8.

80. Kamijo K, Matsui T. Acute extracranial-intracranial bypass using a radial artery graft along with trapping of a ruptured blood blister-like aneurysm of the internal carotid artery. Clinical article. J Neurosurg. 2010; 113: 781-5.

81. Islam MS, Manabe H, Hasegawa S, Takemura A, Nagahata M. Successful staged treatment for ruptured blister-like dissecting aneurysm of the intracranial internal carotid artery: acute GDC embolization for the blister-like aneurysm followed by proximal occlusion with extracranial-intracranial bypass in the chronic stage. Minim Invasive Neurosurg. 2004; 47: 165-8.

82. Cikla U, Baggott C, Baskaya MK. How I do it: treatment of blood blister-like aneurysms of the supraclinoid internal carotid artery by extracranial-to-intracranial bypass and trapping. Acta Neurochir (Wien). 2014; 156: 2071-7.

83. Conrad MD, Brasiliense LB, Richie AN, Hanel RA. Y stenting assisted coiling using a new low profile visible intraluminal support device for wide necked basilar tip aneurysms: a technical report. J Neurointerv Surg. 2014; 6: 296-300. 